\title{
Ginecomastia: revisão de literatura e aspectos clínicos
}

\author{
Gynecomasty: literature review and clinical aspects \\ Ginecomastia: revisión de la literatura y aspectos clínicos
}

Recebido: 09/03/2021 | Revisado: 16/03/2021 | Aceito: 22/03/2021 | Publicado: 30/03/2021

\author{
Luiza La Porta Matos \\ ORCID: https://orcid.org/0000-0003-2311-5719 \\ Universidade do Planalto Catarinense, Brasil \\ E-mail: luizalaporta@uniplaclages.edu.br \\ Ana Lúcia Ravanello de Souza \\ ORCID: https://orcid.org/0000-0001-9984-8981 \\ Universidade do Planalto Catarinense, Brasil \\ E-mail: ana.ravanello@hotmail.com
}

\begin{abstract}
Resumo
Introdução: A ginecomastia é o desenvolvimento benigno da mama masculina. Possui origem fisiológica ou patológica, como consequência de um desequilíbrio entre as quantidades de estrógeno e andrógeno. Pode gerar desconforto, mastalgia, sensibilidade e aumento das mamas. Objetivos: Realizar uma revisão bibliográfica sobre a etiologia da ginecomastia, suas características de apresentação e seu diagnóstico. Método: Foi realizado um levantamento bibliográfico a partir de busca ativa de artigos científicos nas bases de dados PubMed, Scielo e LILACS, com o recorte temporal de 2015 a 2020. A pesquisa foi feita utilizando os descritores (DeCS) gynecomastia, etiology, diagnosis e breast. Nas 3 bases de dados foram encontrados 79 artigos. Desses, foram selecionados 12, que incluem a etiologia da ginecomastia, apresentação e evolução, além do diagnóstico. Foram excluídos artigos que abordavam pseudoginecomastia, relação da ginecomastia com o prognóstico de pacientes com tumores, outras lesões mamárias e efeitos adversos de drogas que causam ginecomastia. Resultados: A faixa-etária mais acometida é entre 50 e 69 anos, com incidência de $72 \%$. A fase puberal é a mais prevalente dos períodos fisiológicos. No período senil, as contribuições para ginecomastia provém do hipogonadismo, diminuição da concentração de testosterona, alteração de LH e valores do estradiol mantidos. A história médica e o exame físico, com a palpação das mamas, são fundamentais para estabelecer o diagnóstico. Conclusão: A ginecomastia é uma circunstância benigna. É necessário diferenciar corretamente entre fisiológica e patológica. $\mathrm{O}$ acompanhamento individualizado, com indicação cirúrgica em alguns casos, é essencial para promover bem-estar e aceitação pessoal.
\end{abstract}

Palavras-chave: Ginecomastia; Etiologia; Diagnóstico; Mama.

\begin{abstract}
Introduction: Gynecomastia is the benign development of the male breast. It has a physiological or pathological origin, as a consequence of an imbalance between the amounts of estrogen and androgen. It can generate discomfort, mastalgia, tenderness and enlargement of the breasts. Objectives: To carry out a literature review on the etiology of gynecomastia, its presentation characteristics and diagnosis. Method: A bibliographic survey was conducted based on an active search for scientific articles in the PubMed, Scielo and LILACS databases, with a time frame from 2015 to 2020. The research was carried out using the descriptors (DeCS) gynecomastia, etiology, diagnosis and breast. In the 3 databases, 79 articles were found. Of these, 12 were selected, which include the etiology of gynecomastia, presentation and evolution, in addition to the diagnosis. Articles that addressed pseudogynecomastia, the relationship between gynecomastia and the prognosis of patients with tumors, other breast lesions and adverse effects of drugs that cause gynecomastia were excluded. Results: The most affected age group is between 50 and 69 years old, with an incidence of $72 \%$. The pubertal phase is the most prevalent of the physiological periods. In the senile period, contributions to gynecomastia come from hypogonadism, decreased testosterone concentration, altered LH and maintained estradiol values. Medical history and physical examination, with palpation of the breasts, are essential to establish the diagnosis. Conclusion: Gynecomastia is a benign condition. It is necessary to differentiate correctly between physiological and pathological. Individualized follow-up, with surgical indication in some cases, is essential to promote well-being and personal acceptance.
\end{abstract}

Keywords: Gynecomastia; Etiology; Diagnosis; Breast.

\section{Resumen}

Introducción: La ginecomastia es el desarrollo benigno de la mama masculina. Tiene un origen fisiológico o patológico, como consecuencia de un desequilibrio entre las cantidades de estrógenos y andrógenos. Puede generar malestar, mastalgia, sensibilidad y agrandamiento de las mamas. Objetivos: Realizar una revisión de la literatura sobre 
la etiología de la ginecomastia, sus características de presentación y diagnóstico. Método: Se realizó un levantamiento bibliográfico basado en una búsqueda activa de artículos científicos en las bases de datos PubMed, Scielo y LILACS, con el marco temporal de 2015 a 2020. La investigación se realizó utilizando los descriptores (DeCS) ginecomastia, etiología, diagnóstico y pecho. En las 3 bases de datos se encontraron 79 artículos. De estos, se seleccionaron 12, que incluyen la etiología de la ginecomastia, presentación y evolución, además del diagnóstico. Se excluyeron los artículos que abordaban la pseudoginecomastia, la relación entre la ginecomastia y el pronóstico de pacientes con tumores, otras lesiones mamarias y los efectos adversos de los fármacos que provocan ginecomastia. Resultados: el grupo de edad más afectado es el de 50 a 69 años, con una incidencia del 72\%. La fase puberal es la más prevalente de los períodos fisiológicos. En el período senil, las contribuciones a la ginecomastia provienen del hipogonadismo, la disminución de la concentración de testosterona, la LH alterada y los valores de estradiol mantenidos. La historia clínica y la exploración física, con palpación de las mamas, son fundamentales para establecer el diagnóstico. Conclusión: la ginecomastia es una condición benigna. Es necesario diferenciar correctamente entre fisiológico y patológico. El seguimiento individualizado, con indicación quirúrgica en algunos casos, es fundamental para promover el bienestar y la aceptación personal.

Palabras clave: Ginecomastia; Etiología; Diagnóstico; Mamá.

\section{Introdução}

A ginecomastia é caracterizada como o desenvolvimento benigno da mama masculina, podendo ser temporária ou definitiva, unilateral ou bilateral. A palavra deriva do grego gyne (mulher) e mastos (mama). Apresenta diversas etiologias que estão subdivididas em 3 grupos, fisiológicos, patológicos e tóxicos (Cachaço, Elias \& Nazário, 2015).

A origem fisiológica é classificada em fases neonatal, puberal e senil, enquanto a patológica provém de causas hormonais, que ocorrem por um desequilíbrio entre as quantidades de estrógeno e andrógeno. É decorrente do avanço da idade, variadas doenças, agentes químicos e drogas, além de possuir fatores idiopáticos que correspondem a $50 \%$ da totalidade de casos (Kanakis et al., 2019).

A maioria das ocorrências é assintomática, porém existem alguns indícios que levam à busca por auxílio médico, como desconforto, mastalgia, sensibilidade e aumento das mamas. O conjunto desses sintomas leva, consequentemente, ao temor de doença maligna pelo paciente. Entretanto, a ginecomastia é determinada por um crescimento benigno do tecido glandular, através de ramificações secundárias dos ductos e hiperplasia estromal, levando ao seu alargamento macio, simétrico e discoide (Cachaço et al., 2015).

Deve ser diferenciada da pseudoginecomastia, condição que ocorre pela amplificação do volume mamário devido ao acúmulo de gordura na obesidade, situação cada vez mais comum na sociedade atual, pelo estilo de vida sedentário. A distinção entre ginecomastia e pseudoginecomastia é feita através da palpação no exame físico ou pela realização de exames de imagem. Ambas as condições podem causar constrangimento e angústia emocional, principalmente nos adolescentes, devido a percepção alterada de sua imagem corporal (Soliman, Sanctis \& Yassin, 2017).

$\mathrm{Na}$ fase da puberdade é normal que a ginecomastia esteja presente nos meninos, com incidência de até $50 \%$, e na grande maioria se resolve naturalmente. Por outro lado, a prevalência de ginecomastia nos adultos é bem diversificada e não se sabe com precisão os seus dados, com estudos apontando variações de 36 a 57\%. A involução espontânea é pouco provável se a permanência da ginecomastia ultrapassar um ano, por conta da fibrose e hialinização (Kanakis et al., 2019).

O objetivo do presente artigo é realizar revisão bibliográfica sobre a etiologia da ginecomastia, suas características de apresentação e seu diagnóstico.

\section{Metodologia}

Para o desenvolvimento do estudo foi realizada revisão de literatura do tipo integrativa, visando reunir achados de trabalhos empíricos e teóricos a fim de sintetizaar resultados e aprofundar a compreensão sobre o tema ginecomastia. A 
Research, Society and Development, v. 10, n. 4, e4310413684, 2021

(CC BY 4.0) | ISSN 2525-3409 | DOI: http://dx.doi.org/10.33448/rsd-v10i4.13684

Revisão integrativa é um método sistematizado de " busca, análise e síntese dos dados, permitindo tomada de decisão frente a determinado problema, a fim de qualificá-lo" (Casarin et al., 2020). Segundo as etapas dessa revisão, foi realizada busca ativa de artigos científicos nas bases de dados PubMed, Scielo e LILACS, com o recorte temporal de 2015 a 2020. A pesquisa foi feita utilizando os descritores (DeCS) gynecomastia, etiology, diagnosis e breast. Para a seleção dos artigos usou-se o operador booleano "AND" com finalidade de restringir a pesquisa aos artigos que apresentavam ao mesmo tempo cada um dos termos.

Nas 3 bases de dados foram encontrados 79 artigos. Desses, foram selecionados 12, que incluem a etiologia da ginecomastia, a sua apresentação e evolução, além do seu diagnóstico. Foram excluídos artigos que abordam pseudoginecomastia, relação da ginecomastia com o prognóstico de pacientes com tumores, outras lesões mamárias, efeitos adversos de drogas que causam ginecomastia ou outras complicações que o homem pode apresentar por alterações hormonais.

\section{Resultados}

O surgimento da propagação secundária dos ductos e a hiperplasia estromal são benignos nos homens e recebem pouca atenção aos estudos, por isso valores epidemiológicos de ginecomastia não são exatos. Contudo, autópsias revelam que 5 a $9 \%$ dos homens têm essa mutação. A adolescência é um grupo muito atingido pela ginecomastia, com prevalência de 30 a $60 \%$, porém a faixa-etária mais acometida fica entre os 50 e 69 anos, com incidência de 72\% (Cachaço et al., 2015).

A etiologia da ginecomastia pode ser compreendida em fisiológica e patológica.

\section{Fisiológica}

A causa fisiológica é estabelecida nas fases neonatal, puberal e senil.

Nos recém-nascidos ocorre em 65-90\%, acredita-se que aconteça pela atuação dos hormônios maternos e placentários (estrogênio, progesterona e peptídeos mamotrópicos). Começa, aproximadamente, três dias após o nascimento e pode durar de 2-3 semanas, com uma resolução espontânea à medida que os hormônios maternos saem da circulação do neonato. Podem retornar durante os primeiros meses de vida na "mini-puberdade", pela ativação do eixo hipotálamo-hipófise-gônadas. Habitualmente sua solução completa ocorre até o primeiro ano de vida (Kanakis et al., 2019).

A fase puberal é a mais prevalente dentre os períodos fisiológicos, atingindo dois em cada três meninos. Inicia-se aos 10 anos, atinge a máxima prevalência aos 14, nos estágios de Tanner 3-4 púbicos, e a regressão se dá em torno dos 17 . Essa condição ocorre pela desregulação na relação androgênio/estrogênio, com uma maior ação dos estrogênios no tecido mamário, o que acarreta em proliferação transitória dos ductos e arredores do tecido mesenquimal. Também ocorre incremento do trabalho da aromatase que converte testosterona e androstenediona para E2 e E1, respectivamente, nos fibroblastos na pele dos adolescentes. Conforme a puberdade avança, os níveis de andrógenos se elevam, ocorrendo involução e atrofia dos ductos (Gottlieb et al., 2015; Lemaine, Cayci, Simmons \& Petty, 2013).

A ginecomastia nos adolescentes é transitória e desaparece em 12 a 18 meses, integralmente nos casos leves. Nas condições moderadas ( $20 \%$ dos acometidos) pode demorar até 3 anos para regredir, enquanto a forma grave ( $8 \%$ dos casos), mais duradoura, pode permanecer por resíduos de fibrose e necessita de tratamento em $40 \%$ dos que a possuem. Visto que a forma leve é a mais comum, o tratamento se faz com o acompanhamento e a observação. Apesar disso, a farmacologia e o tratamento cirúrgico sempre são interrogados, pois essa condição gera grande angústia nos adolescentes, em razão da alteração na percepção da autoimagem (Soliman et al., 2017).

No período senil, as contribuições para ginecomastia provém do hipogonadismo, diminuição da concentração de testosterona, alteração de LH e valores do estradiol mantidos. Além disso, as globulinas ligadoras de hormônios sexuais têm seus índices aumentados, e devido à sua atração pela testosterona diminuem esse hormônio na circulação. A faixa de idade 
Research, Society and Development, v. 10, n. 4, e4310413684, 2021

(CC BY 4.0) | ISSN 2525-3409 | DOI: http://dx.doi.org/10.33448/rsd-v10i4.13684

mais atingida fica entre os 50 e 69 anos. Com o avanço da idade é comum o aumento do IMC, e a prevalência de ginecomastia nos casos em que o IMC está acima de 25 é de $80 \%$, pois a adiposidade contribui para os altos níveis dos estrogênios circulantes. Conforme o paciente vai envelhecendo, seu corpo sofre mudanças estruturais, com o incremento de até $0,4 \mathrm{~kg}$ por ano de gordura. Além disso, o cansaço progressivo impossibilita a realização de atividades físicas, favorecendo o ganho de massa gordurosa e a perda de volume muscular, com gradual ativação da enzima aromatase e aumento de estrógenos (Huang, Rivera, Barrios, Silvestre \& Pérez, 2016).

\section{Patológica}

Cerca de 45-50\% dos portadores de ginecomastia apresentam outra patologia subjacente, como doenças sistêmicas, obesidade e endocrinopatias, incluindo deficiência de testosterona. Seu curso clínico não está totalmente elucidado, mas há indícios de grande relevância entre a desigualdade dos crescentes níveis de estrogênio em relação ao androgênio (Cachaço et al., 2015).

A atividade limitada do andrógeno decorre de um déficit de testosterona ou uma diminuição na afinidade dos seus receptores. A deficiência de testosterona primária resulta de defeito testicular, por orquites, traumas, tumores e seus tratamentos. Essa falha cessará o feedback negativo à hipófise, elevando os níveis de LH, e consequentemente de aromatização. Por outro lado, as causas da deficiência secundária cursam com defeitos genéticos e alterações a nível central, como a liberação reduzida do hormônio liberador de gonadotrofinas (GnRH), LH ou de ambos. Já o aumento de estrógeno provém do excesso de aromatização, com potencial estímulo pelo uso do álcool, excesso de tecido adiposo e estímulo por LH. Desta maneira, como o tecido mamário possui receptores para os dois hormônios, estrogênio atua na estimulação e androgênio na inibição, o resultado final será uma proliferação do tecido (Kanakis et al., 2019).

Além das alterações metabólicas, seu início também pode ocorrer por indução devido ao uso de drogas, que alteram o status do hormônio sexual, representando uma ginecomastia mais complexa e que acomete apenas uma mama. Grande parte dos medicamentos que estão relacionados com a origem da ginecomastia possuem um curso clinico semelhante, com início unilateral de dor/hipersensibilidade, seguindo-se por amplificação da mama. Um dos fármacos mais conhecidos que podem instigar ao desenvolvimento da proeminência mamaria é a espironolactona, mas seus mecanismos ainda são pouco esclarecidos. Diferentemente dos outros fármacos, o mais usual é causar o crescimento bilateral e com ausência de dor. A ginecomastia pode ser consequência do uso por atletas de esteróides anabólicos androgênicos, principalmente, àqueles que possuem andrógenos que aromatizam (Nutall, Warrier \& Gannon, 2015).

Doenças tireoidianas são causas comuns de ginecomastia. O hipertireoidismo está entre as doenças mais relacionadas, manifestando-se em $40 \%$ dos homens que a possuem. Duas correlações são sugeridas, a ação direta dos hormônios tireoidianos sobre a enzima aromatase e o estímulo na produção de globulinas ligadoras de hormônios sexuais, e por conseguinte a diminuição de testosterona livre. Menos comum é a ginecomastia induzida por hipotireoidismo, pode ocorrer por hiperprolactinemia sob estímulo do hormônio liberador da tireóide (TRH), que também irá reduzir os níveis de testosterona circulante. A prolactina também possui receptores no tecido mamário participando no desenvolvimento do tecido. Existem outras causas para hiperprolactinemia, como tumores hipotalâmicos e/ou hipofisários, doenças renais e uso de antipsicóticos (Kanakis et al., 2019).

Uma causa importante de ginecomastia é o alcoolismo, visto que o álcool eleva beta-endorfinas desativadoras da secreção de GnRh, LH e testosterona. Ademais, é uma das principais etiologias da cirrose, que possui associação com a ginecomastia numa prevalência de $44 \%$, já que a insuficiência hepática reduz o catabolismo de estrogênio. Outro motivo da 
Research, Society and Development, v. 10, n. 4, e4310413684, 2021

(CC BY 4.0) | ISSN 2525-3409 | DOI: http://dx.doi.org/10.33448/rsd-v10i4.13684

alta prevalência é que a ascite do paciente cirrótico é tratada com o uso de espironolactona, já descrita como causadora de ginecomastia. (Huang et al., 2016).

Existe correlação entre diferentes tipos de tumores e a ginecomastia. Quando as células de Leyding são acometidas por tumores testiculares benignos verifica-se um incremento nas quantidades de 17b-estradiol. Já o carcinoma de células germinativas libera gonadotrofina coriônica humana (hCG), o que resultará também em aumento de estradiol por estímulo da aromatase. Outro tumor que aumenta os níveis de estrógenos é o carcinoma de adrenais. A causa mais rara de ginecomastia é a síndrome de Kennedy, resultante de alterações genéticas que levam a uma menor sensibilidade do receptor de andrógenos. Por fim, 50\% dos casos não possuem motivos detectáveis, denominando-se de ginecomastia idiopática (Kanakis et al., 2019).

\section{Fases Histológicas}

A ginecomastia estabelece-se com a proliferação dos ductos e suas ramificações, hipervascularização, edema do tecido conjuntivo e do estroma periductal. Esse espiralado hipercelular corresponde a uma etapa que pode ser reversível, desde que o precedente seja tratado. (Huang et al., 2016; Nuttall et al., 2015;). Após um ano de evolução, ocorre a hialinização do estroma e redução do crescimento epitelial, formando uma disposição mais fibrosa com aspirado hipocelular. Esse tecido é resistente ao tratamento e irreversível, principalmente, quando possui dois anos de avanço, desse modo possui indicação cirúrgica (Cachaço et al., 2015).

Sua categorização está dividida em grau I, II e III pelo volume do peito e elasticidade da pele. Grau I consta com aumento de volume da mama e sem sulco submamário, grau II têm-se crescimento do volume com formação de subdobras e grau III ocorre a amplificação e queda da mama, não havendo elasticidade da pele. (Huang et al., 2016).

\section{Diagnóstico}

Predominantemente, os pacientes com ginecomastia são assintomáticos, dando-se o diagnóstico no decorrer do exame físico, muitas vezes por outros motivos de consulta. Para definir ginecomastia é essencial a palpação da massa de tecido mamário subareolar medindo, pelo menos, de 0,5 a $2 \mathrm{~cm}$, por investigadores diferentes. Esse achado equivale a um nódulo discoide e elástico, contrário do que se assemelharia a um câncer, por ter mobilidade e não aderir a planos profundos (Cachaço et al., 2015).

A mama masculina sem alterações encontra-se a $20 \mathrm{~cm}$ do esterno, é mais ou menos plana, com plenitude ao redor do complexo aréola-mamilo. O aparecimento de firmeza incomum, assimetria, secreção mamilar, linfadenopatia axilar e uma massa excêntrica sugerem câncer de mama, nesses casos a mamografia deve ser solicitada. A palpação também deve ser capaz de distinguir uma ginecomastia verdadeira da pseudoginecomastia (Nuttall et al., 2015).

Para aqueles que são sintomáticos, o achado mais significativo é o crescimento da mama, comumente unilateral. Nesses casos a história médica e o exame físico são fundamentais na avaliação. A anamnese deve proceder com detalhes, sobre o princípio da doença, sua duração, sintomatologia, além da busca por fatores de risco. É preciso saber das medicações em uso, doenças concomitantes e sinais de hipogonadismo. Caso seja adolescente, investigar o início e evolução da puberdade. A averiguação na história psicossocial por uso de drogas ou álcool é de grande relevância. A história clínica completa associada ao exame físico muitas vezes é suficiente para o diagnóstico (Cachaço et al., 2015).

Durante a avaliação física devem ser examinados o índice de massa corporal, circunferência da cintura e relação cintura-quadril para mensurar a obesidade (Rivera, Eisenstein \& Cardoso, 2009). O exame inclui a palpação da tireoide, mensuração hepática ou renal. A investigação do desenvolvimento puberal e seus estágios de Tanner devem ser realizados, além da avaliação dos pelos faciais e da massa muscular (Kanakis et al., 2019). 
Deve ser solicitado de rotina os exames laboratoriais: LH (Hormônio Luiteinizante), FSH (Hormônio Foliculoestimulante), T(Testosterona), E² (Estradiol) e SHBG (Globulina ligadora de hormônios sexuais), Beta-HCG (Gonadotrofina coriônica humana), TSH (Hormônio estimulador da tireoide), prolactina, função hepática e renal (Nuttall et al., 2015).

Nos pacientes sem sintomas e cuja história não indica origem patológica o laboratório é dispensável. Concentrações aumentadas de gonadotrofina combinadas com baixas concentrações de T são sugestivas de hipogonadismo primário. Altas concentrações de LH na presença de T normal também pode causar ginecomastia devido ao efeito estimulante de LH na aromatase. Se houver aumento de LH e T, averiguar função tireoidiana. Para valores acima de hCG, a ecografia dos testículos ajuda na identificação de tumores (Cachaço et al., 2015; Kanakis et al., 2019).

Exames de imagem são menos solicitados, feitos em maior quantidade em indivíduos obesos, em razão da maior dificuldade na palpação e diferenciação da pseudoginecomastia (Kanakis et al., 2019). Não há unanimidade sobre qual exame deve ser executado antes, mamografia ou USG, variando de acordo com os padrões de sensibilidade e especificidade (Nuttall et al., 2015).

\section{Discussão}

Diante da análise dos resultados, observou-se que a literatura foi unânime quanto ao alto desenvolvimento da ginecomastia com o passar da idade. A progressão está relacionada com o hipogonadismo, aumento da aromatização dos androgênios em estrogênios e a mudança conformacional do corpo, com gradual aumento de massa gordurosa. Assim, em jovens e homens adultos (17-58 anos) a ginecomastia corresponde a 36\%, já em homens com mais de 44 anos a porcentagem sobe para 50\%. Pacientes hospitalizados, com idades entre 58 a 71 anos, a prevalência é em 65\% (Nuttall et al., 2015).

Em relação às etiologias patológicas, o grupo mais expressivo foi por causas idiopáticas, com 47,8\%. As outras causas mais frequentes são ginecomastia puberal anabólica, com 7,8\% dos pacientes. Além disso, é levado em conta o hipogonadismo e o uso de drogas como causas proeminentes (Oranierc \& Zgliczynski, 2008).

Pode-se perceber que a ginecomastia puberal, de caráter fisiológico, regride espontaneamente em 1 a 3 anos (Soliman et al., 2017). Apesar disso, se o diâmetro for maior do que $2 \mathrm{~cm}$, poderá persistir, dificultando a involução, pois aumentam as chances de fibrose residual. A regressão da ginecomastia patológica, com mais de dois anos de evolução também se torna comprometida pelo mesmo processo de fibrose e hialinização (Cachaço et al., 2015).

Na maior parte dos casos não será necessário realizar exame de imagem. A sua indicação é feita quando se suspeita de malignidade, a partir de massas excêntricas palpáveis ao exame físico, nesses casos a mamografia deve ser solicitada. No entanto, quando a suspeita ainda é de ginecomastia, mas precisa de um exame de imagem para confirmar, a literatura possui divergência quanto ao exame de imagem a ser requisitado. Alguns preferem o ultrassom, por seu menor custo e facilidade de execução em homens. Já outros defendem que a mamografia é o melhor exame, por sua maior sensibilidade (Cachaço et al., 2015).

O seguimento laboratorial também teve divergências na literatura, visto que a avaliação hormonal permanece controversa. Estudos apontam que os exames laboratoriais serão solicitados quando não caracterizarem fases fisiológicas, houverem incertezas do diagnóstico, além de casos em que o crescimento das mamas ocorreu de forma muito rápida. Pacientes com ginecomastia assintomáticos e sem suspeita de patologia subjacente podem somente ficar em observação (García, Jiménez, Lozano \& Moraño, 2018). Sob outra perspectiva, alguns autores acreditam que qualquer ginecomastia que ocorra fora das fases fisiológicas deve ter sua avaliação laboratorial realizada (Ukaonu, JR \& Curtis, 2017). O laboratório clínico pode 
incluir, quando solicitado, testes de função renal e hepática, TSH, testosterona, estradiol, LH, FSH, prolactina e Beta-HCG (Pusl \& Stormmer, 2017; Wang, Yu, Zhu \& Zeng, 2019).

O tratamento cirúrgico é indicado aos indivíduos que não apresentaram melhora com o tratamento clínico e também aos que evoluíram para a ginecomastia irreversível. Entretanto, em muitos estudos foram abordados o desejo dos pacientes, principalmente dos adolescentes, em serem submetidos a um procedimento cirúrgico, por conta do aspecto estético. Nesses casos, precisa-se ampliar o olhar ao psíquico do paciente, quando o impacto em suas vidas for grande a ponto de repercutir em quadros de ansiedades, depressão e isolamentos sociais, a opção cirúrgica é repensada (Kanakis et al., 2019). Apesar disso, o tecido mais encontrado nos centros cirúrgicos é de aspecto fibrótico, o que caracteriza um predomínio nas salas de operações de ginecomastia de longa duração e irreversível (Blau, Hazani \& Hekma, 2016).

\section{Conclusão}

A ginecomastia é uma circunstância benigna, em que ocorre o desenvolvimento da mama masculina. Pode ser fisiológica, com regressão espontânea, na grande maioria das vezes ou patológica, por um desequilíbrio entre os níveis de estrógeno e andrógeno. Para uma diferenciação correta é necessário detectar a etiologia de base, a partir de uma boa história clínica e exames laboratoriais, quando necessários.

O paciente nessa circunstância, seja de forma fisiológica ou patológica, possui dificuldade em lidar com a condição, principalmente os adolescentes, visto que há uma alteração da sua autoimagem, o que pode repercutir em ansiedade, depressão e isolamento social. Portanto, o acompanhamento individualizado, com indicação cirúrgica em alguns casos, é essencial para promover bem-estar e aceitação pessoal.

Baseado no estudo realizado, notou-se a necessidade de continuidade deste trabalho, abordando as características dos possíveis tratamentos. Deste modo, propiciará maior abrangência do conhecimento sobre a temática abordada, contribuindo para a qualidade de vida e bem-estar dos homens acometidos.

\section{Referências}

Blau, M., Hazani, R., \& Hekma, D. (2016). Anatomy of the gynecomastia tissue and its clinical significance. Plast Reconstr Surg Glob Open, 4(8), e854. https://www.ncbi.nlm.nih.gov/pmc/articles/PMC5010345/

Cachaço, E. E., Elias, S., \& Nazário, A. C. P. (2015). Ginecomastia. Femina, 43(5), 197-202. http://files.bvs.br/upload/S/0100-7254/2015/v43n5/a5315.pdf

Casarin, S. T., Porto, A. R., Gabatz, R. I. B., Bonow, C. A., Ribeiro, J. P., \& Mota, M. S. (2020). Tipos de revisão de literatura: considerações das editoras do Journal of Nursing and Health. J. nurs. Health, 10(5), e20104031. https://periodicos.ufpel.edu.br/ojs2/index.php/enfermagem/article/view/19924

Costanzo, P. R., Suárez, S., Aszpis, S. M., Usher, S. J. G., Pragier, U. M., \& Cavoia, V. M., et al. (2015). Ginecomastia: aspectos clínicos y etiológicos estudio retrospectivo y multicêntrico. Revista Argentina de Endocrinología y Metabolismo, 52(1), 22-28. http://www.scielo.org.ar/scielo.php?script=sci_arttext\&pid=S851-30342015000100004

García, C. P., Jiménez, A. B. A., Lozano, L. C., \& Moraño, C. C. (2018). Importancia de la anamnesis exhaustiva ante una ginecomastia unilateral inexplicada en un niño. Arch Argent Pediatr, 116(5), 655-658. https://www.sap.org.ar/docs/publicaciones/archivosarg/2018/v116n5a15.pdf

Gottlieb, S., Pasqualini, T., Martínez, A., Bengolea, S. V., Azaretzky, M., \& Ciaccio, M., et al. (2015). Características clínicas y etiología de la ginecomastia en pacientes en edad prepuberal. Revista Argentina de Endocrinología y Metabolismo, 52(2), 57-65. http://www.raem.org.ar/numeros/2015-vol52/numero02/57-65-endo2-3-gottlieb.pdf

Huang, S. H., Rivera, J., Barrios, M. C., Silvestre, R., \& Pérez, R. G. (2016). Ginecomastia de origen multifactorial en el adulto mayor. revisión fisiopatológica de un caso. Revista Venezolana de Endocrinología y Metabolismo, 14(1), 45-55. http://ve.scielo.org/scielo.php?script=sci_arttext\&pid=S169031102016000100006

Kanakis, G. A., Nordkap, L., Bang, A. K., Calogero, A. E., Bartfai, G. A., \& Corona, G., et al. (2019). EAA clinical practice guidelines- gynecomastia evaluation and management. Andrology, 7(6), 778-793. https://pubmed.ncbi.nlm.nih.gov/31099174/

Lemaine, V., Cayci, C., Simmons, P. S. \& Petty, P. (2013). Gynecomastia in Adolescent Males. Guest Editors, 27(1), 56-61. https://www.ncbi.nlm.nih.gov/pmc/articles/PMC3706045/ 
Research, Society and Development, v. 10, n. 4, e4310413684, 2021

(CC BY 4.0) | ISSN 2525-3409 | DOI: http://dx.doi.org/10.33448/rsd-v10i4.13684

Nuttall, F. Q., Warrieri, R. S., \& Gannon, M. C. (2015). Gynecomastia and drugs: a critical evaluation of the literature. Eur J Clin Pharmacol, 71, 569-578. https://link.springer.com/article/10.1007/s00228-015-1835-x

Oraniec, I. C., \& Zgliczynski, W. (2008). Phenotype of patients with gynecomastia. Endokrynol Pol, 59(2), 131-139. https://pubmed.ncbi.nlm.nih.gov/18465688/

Pusl, T., \& Stoemmer, P. (2017). Gynecomastia: look beyond the obvious. The American Journal of Medicine, 130(10), 439-440. https://www.amjmed.com/action/showPdf?pii=S0002-9343\%2817\%2930489-8

Riveral, N. F., Eisenstein, E., \& Cardoso, C. B. M. A. (2008). Relação da ginecomastia puberal com o índice de massa corporal. Arq Bras Endocrinol Metab, 5(1), 23-28. https://www.scielo.br/pdf/abem/v53n4/v53n4a08.pdf

Soliman, A. T., Sanctis, V., \& Yassin, M. (2017). Management of adolescent gynecomastia: an update. Acta bio-medica: Atenei Parmensis, 88(2), $204-213$. https://www.ncbi.nlm.nih.gov/pmc/articles/PMC6166145/

Ukaonu, C. E., JR, G. M. C., \& Curtis, S. A. (2017). Severe male breast enlargement. Am Fam Physician, 95(9), 583-584. https://pubmed.ncbi.nlm.nih.gov/28671390/

Wang, C., Yu, N., Zhu, L., \& Zeng, A. (2019). Idiopathic prepubertal unilateral gynecomastia. Clinical Case Report, 98(40), e17374. https://www.ncbi.nlm.nih.gov/pmc/articles/PMC6783188/ 